\title{
Reliability Analysis of a Standby Redundant System with Critical Human Error
}

\author{
Digvijay Singh \\ Dept. of Mathematics \\ J.P.Institute of Engg. \& Tech. \\ Meerut(U.P)
}

\author{
Viresh Sharma \\ Dept. of Mathematics \\ N.A.S.(P.G.) College \\ Meerut (U.P.)
}

\author{
Smita Sharma \\ Dept. of Statistics \\ D.A.V.(P.G.) College \\ Dehradun (U.K.)
}

\begin{abstract}
The author has considered a transient system composed of two indentical units in a standby mode which can fail due to hardware common cause failure. Initially, one unit is operational mode and other in standby mode. The operative unit may fail partially or totally. In case of total failure of first unit, standby unit becomes operational system works with full efficiency.
\end{abstract}

\section{INTRODUCTION}

In this paper, the author has considered a transient system composed of two identical units in a standby mode which can fail due to hardware common cause failure. Initially, one unit is operational mode and other in standby mode. The operative unit may fail partially or totally. In case of total failure of first unit, standby unit becomes operational system works with full efficiency. It is assumed that whenever there is a hardware failure, system goes partially or totally failure mode first and then total failure of either unit, system works with lesser efficiency. System may fail either due to failure of both units and common cause failure. The entire system can also fail due to critical human errors. The system can be repaired in degraded state, or in failed state due to the unit failures, but can not be repaired when it fails due to human errors.

\section{NOTATIONS}

(a)

$D / D t / D x / D y / D z / D w: \frac{d}{d t} / \frac{\partial}{\partial t} / \frac{\partial}{\partial x} / \frac{\partial}{\partial y} / \frac{\partial}{\partial z} / \frac{\partial}{\partial w}$

(b)

$$
\alpha_{1} / \alpha_{2} / \alpha_{3} / \alpha_{4}: \begin{gathered}
\text { Constant failure due to } \\
\text { minor/major errors, }
\end{gathered}
$$

(c) $\lambda_{h G} / \lambda_{h D}$ : Constant failure rate due to critical/human errors when the system is in good state,
(d) $\boldsymbol{P}_{\mathrm{O} s} \boldsymbol{t}: \boldsymbol{P}:$ (at time t the system is in state

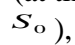
(e) $P_{P s} t(x, t) \square: P: \quad$ (the system is in state $S_{1}$ at time $\mathrm{t}$ due to minor failure and repair time lies in the interval $(x, x+\square)$,
(f) $\quad P_{T 0} t(y, t) \square: P$ : (the system in in state $S_{2}$ at time $t$ and elapsed repair time lies in the interval

$$
(y, y+\square) \text {, }
$$
(g) $\quad P_{T P} t(z, t) \square: P:$ (the system is in the state $S_{3}$ at time $t$ and elapsed repair time lies in interval $(z, z+\square)$,
(h) $P_{T T} t(w, t) \square: P$ : (the system is state $S_{4}$ at time $t$ and elapsed repair time lies in the interval $(w, w+\square)$,
(i) $P_{C}(x, t) \square: P$ : (the system is in state $S_{5}$ at time $\mathrm{t}$ and elapsed repair time lies in interval $(x, x+\square)$

$$
S_{i}^{K}(s)=K(x) e^{-\int_{0}^{x} K x d x}, \quad \text { where }
$$$$
K=\beta_{1}, \psi_{1}, \phi_{1}, \phi_{2}, w_{c}
$$

$\sum_{i}=\sum_{i=1}^{n}$, , unless otherwise stated. 

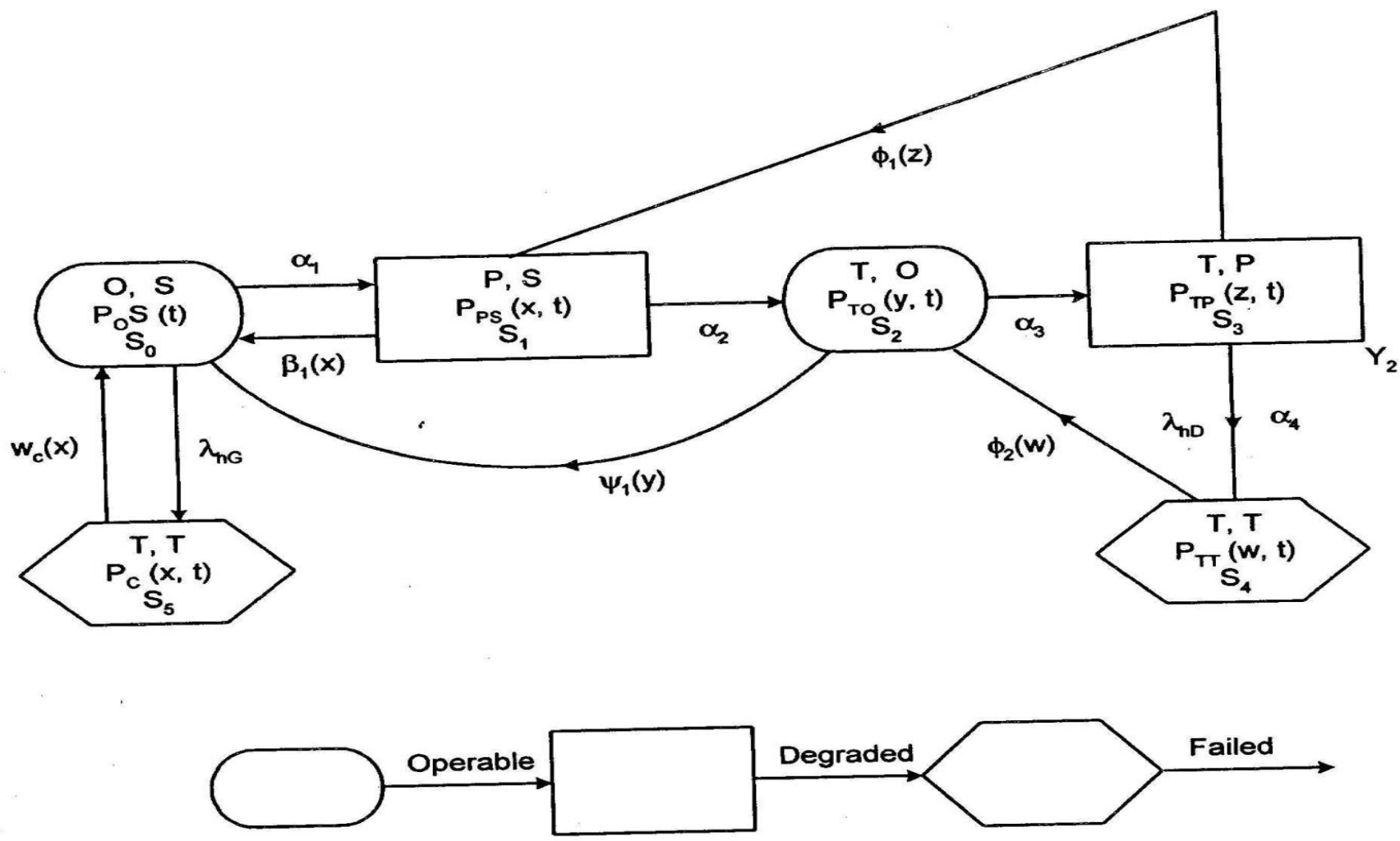

Fig 1: Transition diagram

\section{FORMULAION OF THE}

\section{MATHEMATICAL PROBLEM}

Viewing the nature of this problem the following set of difference differential equations is obtained.

$\left(D+\alpha_{1}+\lambda_{h G}\right) P_{o s}^{(t)}=\int \beta_{1}(x) P_{P S}(x, t) d x$

$+\int w_{C}(x) P_{C}(x, t) d x+\int \psi_{1}(y) P_{T 0}(y, t) d y(1)$

$\left[D x+D t+\alpha_{2}+\beta_{1}(x)\right] P_{P S}(x, t)=\mathrm{O}_{(2)}$

$\left[D y+D t+\alpha_{3}+\psi_{1}(y)\right] P_{T 0}(y, t)=0$ (3)

$\left[D z+D t+\alpha_{4}+\phi_{1}(z)+\lambda_{h D}\right] P_{T P}(z, t)=0$ (4)

$\left[D w+D t+\phi_{2}(w)\right] P_{T T}(w, t)=\lambda_{h D} P_{T P}(z, t)(5)$

$\left[D x+D t+w_{C}(x)\right] P_{C}(x, t)=\lambda_{h G} P_{0 S}(t)$

\section{BOUNDARY CONDITIONS}

$P_{P S}(0, t)=\alpha_{1} P_{0 s}(t)+\int \phi_{1}(z) P_{T P}(z, t) d z \quad$ (7) $P_{T 0}(0, t)=\alpha_{2} \int P_{P_{s}}(x, t) d t+\int \phi_{2}(w) P_{T T}(w, t) d w(8)$ $\boldsymbol{P}_{T P}(\mathrm{O}, \boldsymbol{t})=\alpha_{3} \int \boldsymbol{P}_{T \mathrm{O}}(y, t) d y{ }_{(9)}$ $P_{T T}(\mathrm{O}, t)=\left(\lambda_{h D}+\alpha_{4}\right) P_{T P}(z, t)(10)$

$P_{C}(\mathrm{O}, t)=\lambda_{h G} P_{0 s}(t)$

\section{INITIAL CONDITION:}

$$
P_{0 S}(0)=1, \quad \text { otherwise } 0 \text {. }
$$

SOLUTION OF THE PROBLEM: Taking laplace transform of equations (1) through (11) and using initial conditions, one may obtain

$$
\begin{aligned}
& \left(s+\alpha_{1}+\lambda_{h G}\right) P_{0 S}^{*}(s)=1+\int \beta_{1}(x) P_{P S}^{*}(x, s) d x \\
& +\int w_{C}(x) P_{C}^{*}(x, s) d x+\int \psi_{1}(y) P_{T 0}^{*}(y, s) d y, \\
& {\left[D x+s+\alpha_{2}+\beta_{1}(x) P_{P S}^{*}(x, s)=0,\right. \text { (12) }} \\
& {\left[D y+s+\alpha_{3}+\psi_{1}(y)\right] P_{T 0}^{*}(y, s)=0, \text { (14) }} \\
& {\left[D z+s+\alpha_{4}+\phi_{1}(z)+\lambda_{h D}\right] P_{T P}^{*}(z, s)=0,} \\
& {\left[D w+s+\phi_{2}(w)\right] P_{T T}^{*}(w, s)=P_{T P}^{*}(z, s)\left(\lambda_{h D}+\alpha_{4}\right),(16)} \\
& {\left[D x+s+w_{c}(x)\right] P_{C}^{*}(x, s)=\lambda_{h G} P_{0 s}^{*}(s),} \\
& P_{P s}^{*}(0, s)=\alpha_{1} P_{0 s}^{*}(s)+\int \phi_{1}(z) P_{T P}^{*}(z, s) d z, \\
& P_{T 0}^{*}(0, s)=\alpha_{2} \int P_{P s}^{*}(x, s) d s+\int \phi_{2}(w) P_{T T}^{*}(w, s) d w,(19) \\
& \boldsymbol{P}_{T P}^{*}(\mathbf{O}, s)=\boldsymbol{\alpha}_{3} \int \boldsymbol{P}_{T 0}^{*}(y, s) d y, \text { (20) }
\end{aligned}
$$


$P_{T T}^{*}(\mathrm{O}, s)=\left(\lambda_{h D}+\alpha_{4}\right) P_{T P}^{*}(z, s)$, (21)

$$
P_{C}^{*}(\mathrm{O}, s)=\lambda_{h G} P_{\mathrm{O} s}^{*}(s) \cdot(22)
$$

\section{Integrating the above equations, we}

\section{obtain}

$P_{P S}^{*}(x, s)=\left(\alpha_{1} P_{0 s}^{*}(s)+\int \phi_{1}(z) P_{T P}^{*}(z, s) d z\right) e^{-\left(s+\alpha_{2}\right) x} e^{-\int_{0}^{x} \beta_{1}(x) d x},(23)$

$P_{T 0}^{*}(y, s)=\left(\alpha_{2} \int P_{P_{s}}^{*}(x, s) d x+\int \phi_{2}(w) P_{T T}^{*}(w, s) d w\right)(24)$

$P_{T P}^{*}(z, s) \square\left(\alpha_{3} \int P_{T 0}^{*}(y, s) d y\right) e^{-z\left(s+\lambda_{h G}\right)} e^{-\int_{0}^{z} \phi_{1}(z) d z}(25)$

$P_{C}^{*}(x, s) \square \quad \lambda_{h G} P_{0 s}^{*}(s) e^{-s x} e^{-\int_{0}^{x} w c(x) d x}$ (26)

$P_{T T}^{*}(w, s) \square e^{-s w} e^{-\int_{0}^{w} \phi_{2} w d w}\left(\alpha_{4}+\lambda_{h D}\right) P_{T P}^{*}(z, s)(27)$

$\left(\mathrm{s}+\alpha_{1}+\lambda_{\mathrm{hG}}\right) \mathrm{P}_{0 \mathrm{~S}}^{*}(\mathrm{~s})=1+\left[\alpha_{1} \mathrm{P}_{\mathrm{OS}}^{*}(\mathrm{~s})+\int \phi_{1}(\mathrm{z}) \mathrm{P}_{\mathrm{TP}}^{*}(\mathrm{z}, \mathrm{s}) \mathrm{dz}\right]$

$\int \beta_{1}(x) e^{-\left(s+\alpha_{2}\right) x} \cdot e^{-\int_{0}^{x} \beta_{1}(x) d x}+\lambda_{h G} P_{0 S}^{*}(s) \int w_{c}(x) d x e^{-s(x)} e^{-\int_{0}^{x} w_{c}(x) d x}$

$+\left(\alpha_{2} \int P_{P S}^{*}(x, s) d x+\int \phi_{2}(w) P_{T T}^{*}(w, s) d w\right) \int \psi_{1}(y) d y \cdot e^{-\left(s+\alpha_{3}\right) y} \cdot e^{-\int \psi_{1}(y) d y}$

On solving above equation, we fin $d$

$$
\begin{aligned}
& \bar{P}_{0 s}(s)=\frac{1}{D(s)}, \\
& \bar{P}_{P S}(s)=\frac{B(s)}{D(s)},
\end{aligned}
$$

$\bar{P}_{T 0}(s)=\frac{\alpha_{1} \alpha_{2}}{A(s)} \cdot \frac{1-\bar{S}_{\beta_{1}}\left(s+\alpha_{2}\right)}{s+\alpha_{2}} \cdot \frac{1-\bar{S}_{\psi_{1}}\left(s+\alpha_{3}\right)}{s+\alpha_{3}} \cdot \frac{1}{D(s)}$,

$\bar{P}_{T P}(s)=\frac{C(s)}{D(s)}$,

$\bar{P}_{T T}(s)=\left(\lambda_{h D}+\alpha_{4}\right) \frac{1-\bar{S}_{\phi_{2}}(s)}{s} \frac{C(s)}{D(s)}$,

$\bar{P}_{C}(s)=\lambda_{h G} \frac{1-\bar{S}_{w c}(s)}{s} \frac{1}{D(s)}$.
Evaluation of Laplace transforms of up and down state Probabilities.

$$
\begin{aligned}
& \bar{P}_{u p}(s)=\bar{P}_{0 s}+\bar{P}_{P S}(s)+\bar{P}_{T 0}(s)+\bar{P}_{T P}(s) \\
& =\left[1+\beta(s)+\frac{\alpha_{1} \alpha_{2}}{A(s)} \cdot \frac{1-\bar{S}_{\beta_{1}}\left(s+\alpha_{2}\right)}{s+\alpha_{2}} \cdot \frac{1-\bar{S}_{\psi_{1}}\left(s+\alpha_{3}\right)}{s+\alpha_{3}}\right] \frac{1}{D(s)} . \\
& \bar{P}_{\text {down }}(s)=\bar{P}_{T T}(s)+\bar{P}_{C}(s) \\
& =\left[\left(\lambda_{h D}+\alpha_{4}\right) C(s) \frac{1-\bar{S}_{\phi_{2}}(s)}{s}+\lambda_{C} \frac{1-\bar{S}_{w c}(s)}{s}\right] \frac{1}{D(s)} .
\end{aligned}
$$

It is worth noticing that

$$
\bar{P}_{u p}(s)+\bar{P}_{d o w n}(s)=1
$$

\subsection{Particular Cases}

When repair follows exponential time distribution setting :

$$
\bar{S}^{\theta}(s) \frac{\theta}{(s+\theta)}
$$

Where $\theta=\psi_{1}, \phi_{1}, \beta_{1}, \phi_{2}, w_{c}$

$$
\begin{aligned}
\bar{P}_{o s}(s) & =\frac{1}{E(s)}, \\
\bar{P}_{P s}(s) & =\frac{F(s)}{E(s)}, \\
\bar{P}_{T 0}(s)= & \frac{1}{G(s)} \alpha_{1} \alpha_{2} \frac{1}{s+\alpha_{2}+\beta_{1}} \frac{1}{s+\alpha_{3}+\psi_{1}} \cdot \frac{1}{E(s)}, \\
& \bar{P}_{T P}(s)=\frac{H(s)}{E(s)}, \\
& \bar{P}_{T T}(s)=\frac{H(s)}{E(s)}, \\
& \bar{P}_{T T}(s)=\left(\alpha_{4}+\lambda_{h D}\right) \cdot \frac{1}{s+\phi_{2}} \cdot \frac{H(s)}{E(s)}, \\
& \bar{P}_{C}(s)=\frac{\lambda_{C}}{s+w_{C}} \frac{1}{E(s)} . \\
& M T T F=\int_{0}^{\infty} R(t) d t \\
= & {\left[\frac{A}{\alpha_{1}+y_{c}}+\frac{B}{\alpha_{2}}+\frac{C}{\alpha_{3}}+\frac{D}{\alpha_{4}+\lambda_{h D}}\right] . }
\end{aligned}
$$




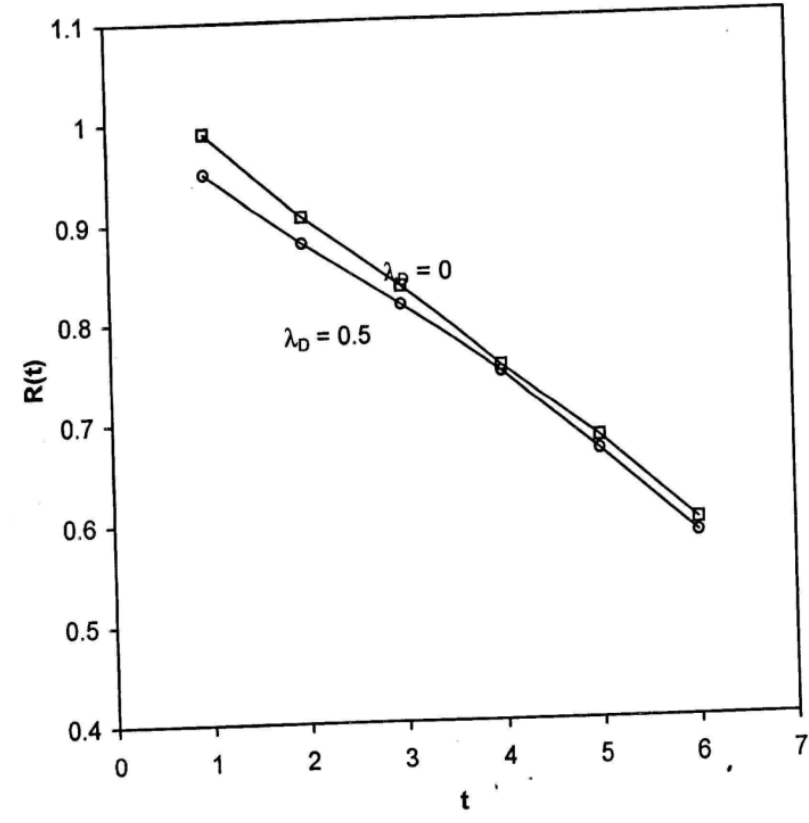

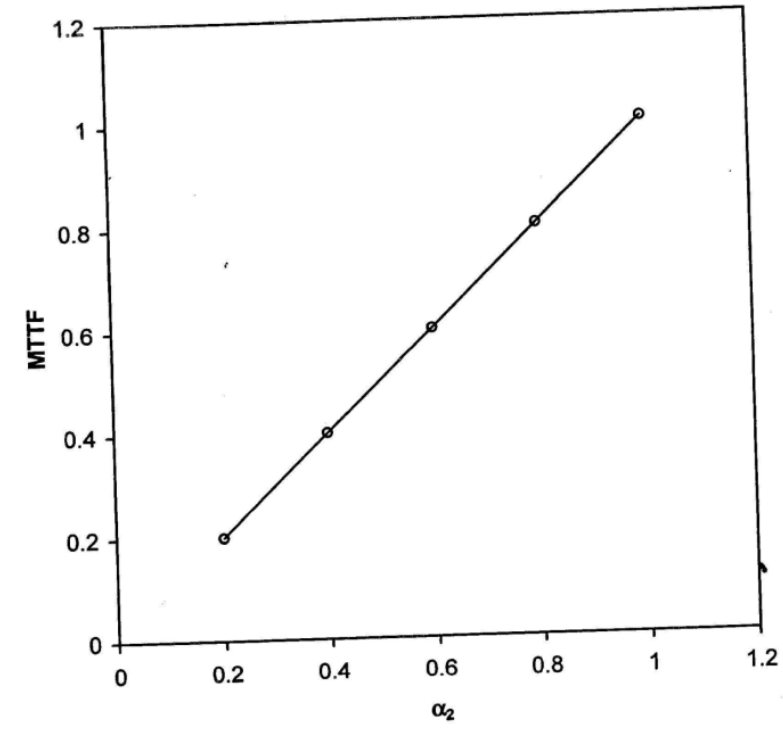

INTERPRETATION :

\section{NUMERICAL COMPUTATIONS :}

$\alpha_{1}=0.1, \alpha_{2}=0.2, \alpha_{3}=0.3, \alpha_{4}=0.4, \lambda_{c}=0.05, \lambda_{D}=0.05 / 0.00$,

$$
\begin{gathered}
\phi_{1}=\psi_{1}=\phi_{2}=\beta_{1}=w_{c}=1 . \\
A=8.32 / 8.9, \\
B=-10.8 / 12, \\
C=4 / 5.33, \\
D=-0.55 /-1.2 .
\end{gathered}
$$

(a) Fig. (1) shows that reliability decreases with increase in time and also effect of $\lambda_{h D}$ also causes a decrease in reliability.

(b) Fig. (2) shows that MTTF decrease for different values of error.

\section{REFERENCES}

[1] Bowman, M., Debray, S. K., and Peterson, L. L. 1993. Reasoning about naming systems. .

[2] Ding, W. and Marchionini, G. 1997 A Study on Video Browsing Strategies. Technical Report. University of Maryland at College Park.

[3] Fröhlich, B. and Plate, J. 2000. The cubic mouse: a new device for three-dimensional input. In Proceedings of the SIGCHI Conference on Human Factors in Computing Systems

[4] Tavel, P. 2007 Modeling and Simulation Design. AK Peters Ltd.

[5] Sannella, M. J. 1994 Constraint Satisfaction and Debugging for Interactive User Interfaces. Doctoral Thesis. UMI Order Number: UMI Order No. GAX9509398., University of Washington.

[6] Forman, G. 2003. An extensive empirical study of feature selection metrics for text classification. J. Mach. Learn. Res. 3 (Mar. 2003), 1289-1305.

[7] Brown, L. D., Hua, H., and Gao, C. 2003. A widget framework for augmented interaction in SCAPE.

[8] Y.T. Yu, M.F. Lau, "A comparison of MC/DC, MUMCUT and several other coverage criteria for logical decisions", Journal of Systems and Software, 2005, in press.

[9] Spector, A. Z. 1989. Achieving application requirements. In Distributed Systems, S. Mullender 